\title{
Negative Charge Management to Make Fragile Bonds No Longer Fragile towards Electrons for Robust Organic Optoelectronic Materials
}

\author{
Rui Wang, ${ }^{[a]}$ Qing-Yu Meng, ${ }^{[a]}$ Yi-Lei Wang, ${ }^{[a]}$ and Juan Qiao*[a,b] \\ [a] R. Wang, Q.-Y. Meng, Dr. Y.-L. Wang, Dr. J. Qiao \\ Key Lab of Organic Optoelectronics and Molecular Engineering of Ministry of Education, Department of Chemistry, Tsinghua University, \\ Shuangqing Road 30, Haidian District, Beijing 100084 \\ Beijing (P. R. China) \\ E-mail: qjuan@mail.tsinghua.edu.cn \\ [b] Dr. J. Qiao \\ Center for Flexible Electronics Technology, Tsinghua University \\ Shuangqing Road 30, Haidian District, Beijing 100084 \\ Beijing (P. R. China) \\ Supporting information for this article is given via a link at the end of the document.
}

\begin{abstract}
The development of robust organic (opto)electronic devices is mainly depressed by the poor intrinsic stability of organic materials on service. For organic light-emitting diode (OLED) materials, a key parameter for intrinsic stability is the bond-dissociation energy of the most fragile bond $\left(\mathrm{BDE}_{\mathrm{f}}\right)$. Although rarely concerned, many OLED molecules have the lowest $\operatorname{BDE}_{\mathrm{f}}$ in anionic states $\left(\mathrm{BDE}_{\mathrm{f}}(-) \sim 1.6-2.5 \mathrm{eV}\right)$, which could be a fatal short-slab for device stability. Here, we confirmed the clear relationship between $\operatorname{BDE}_{\mathrm{f}}(-)$, intrinsic material stability, and device lifetime, and further developed a general and effective strategy to promote $\operatorname{BDE}_{\mathrm{f}}(-) \sim 1 \mathrm{eV}$ for various fragile bonds by introducing appropriate negative charge manager within the molecule. The manager can firmly confine negative-charge and protect fragile bonds, which was verified in typical phosphine-oxide and carbazole derivatives, and backed by newly-designed molecules. This tactic provides a new perspective to reform the vulnerable building blocks for robust organic (opto)electronic materials and devices.
\end{abstract}

\section{Introduction}

The operational stability is a crucial and common issue for organic (opto)electronic devices. ${ }^{[1]}$ In particular for organic light-emitting diodes (OLEDs), which have become the popular display for mobile phones, wearables, TVs, and VR headsets in recent years, the operational stability is still one of the greatest remaining impediment for next-generation display and lighting technology. The intrinsic degradation of OLEDs is mainly ascribed to the chemical deterioration of organic (or metal-organic) materials. ${ }^{[1 \mathrm{~d}, 2]}$ Many (photo)physical processes could induce such chemical deterioration, like exciton-polaron and exciton-exciton annihilations (EPA and EEA), in which EPA has been confirmed as a dominate mechanism. ${ }^{[3]}$ In that process (Figure 1a), one exciton transfers energy to a polaron, generating an excited polaron whose energy could be high enough to break chemical bonds and incur chemical deteriorations. In past decades, considerable efforts have been made to suppress those unwanted (photo)physical processes. ${ }^{[3 a, 4]}$ However, completely avoiding them at the microscopic level is scarcely possible, while even a small amount of deterioration products can result in $50 \%$ luminance loss. ${ }^{[3 a, 5]}$ Therefore, restraining the induced (photo)chemical deteriorations is essential.

According to thermodynamics, the bond most probable to break would be the fragile bond with the minimum (or comparable-to-theminimum) bond-dissociation energy $(\mathrm{BDE})$ in that molecule. Its $\mathrm{BDE}\left(\mathrm{BDE}_{\mathrm{f}}\right)$ has been confirmed as a key parameter for the intrinsic stability of OLED materials by mounting evidences ${ }^{[6]}$ In general, the chemical bonds of organic molecules are particularly vulnerable in anionic states. In Figure $1 \mathrm{~b}$ and $1 \mathrm{c}$, we listed the $\operatorname{BDE}(\mathrm{n}), \operatorname{BDE}(+)$, and $\operatorname{BDE}(-)(n,+$, and - refer to neutral, cationic and anionic states) values of typical fragile exocyclic $C-X$ single bonds $(X=$ heteroatoms like $N, P, S$, etc.) in several representative OLED molecules. It can be found that the $\operatorname{BDE}_{f}(n)$ and $\mathrm{BDE}_{f}(+)$ of most interested molecules are 3.1-4.8 eV, while most $\mathrm{BDE}_{\mathrm{f}}(-)$ are only 1.6-2.5 eV. Many OLED molecules have been reported with comparable $\mathrm{BDE}_{\mathrm{f}}$ values. ${ }^{[2 \mathrm{~b}, 6]}$ As a result, once such negative polarons are generated and/or get involved in EPA, the fragile bonds would be apt to dissociate and incur chemical deterioration. Therefore, $\mathrm{BDE}_{\mathrm{f}}(-)$ would be a fatal short-slab of the intrinsic stability for OLED materials, and deserves a special focus in the study on the related material and device degradation.

The intrinsic degradation of OLED materials in anionic states was first reported by Aziz et al. in tris(8-hydroxyquinoline) aluminum $\left(A l q_{3}\right)$-based devices. ${ }^{[7]}$ They found that excessive electrons in $\mathrm{Alq}_{3}$ layer could form electron traps and incur the efficiency loss of the devices. For OLED molecules, we first concerned the BDE values of charged states in the study of phosphine-oxide materials, ${ }^{[6 \mathrm{~d}]}$ and found that the typical host $\mathrm{CzPO} 2$, whose $\mathrm{BDE}_{\mathrm{f}}(-)$ is only $2.15 \mathrm{eV}$, showed serious $\mathrm{C}-\mathrm{P}$ cleavage in electron-only devices. Since then, a cognition has been accepted that PO could undermine the device stability due to the fragile C-P bonds. ${ }^{[2 b, 66,6 k, 8]}$ Later, Lee et al. found the carbazole $(\mathrm{Cz})$-based host with higher $\mathrm{BDE}$ in charged states contributed to longer device lifetime. ${ }^{[9 a]}$ Recently, more explores have demonstrated prolonged device lifetime based on the consideration of BDE(-). ${ }^{\left[{ }^{9]}\right.}$ Nevertheless, studies on the regulation of BDE(-) for organics are mainly about organic halides. ${ }^{[10]}$ For OLED molecules, Brédas et al. ${ }^{[6 j]}$ recently looked into the substituent effects of the 
cyano, fluorine, and hydroxyl groups on the $\mathrm{BDE}(-)$ values of the $\mathrm{C}-\mathrm{N}$ bonds in the $\mathrm{Cz}$-dibenzothiophene derivatives. However, it still remains largely elusive about the rational regulation of $\mathrm{BDE}_{\mathrm{f}}(-)$ for various OLED molecules.

Different from $\operatorname{BDE}_{f}(n)$ which mainly depends on the bond type, $\operatorname{BDE}_{f}(-)$ is largely affected by the whole molecular structure, as evidenced by the almost identical $\mathrm{BDE}_{\mathrm{f}}(\mathrm{n})$ and very different $\mathrm{BDE}_{\mathrm{f}}(-)$ of the same $\mathrm{C}-\mathrm{N}$ or $\mathrm{C}-\mathrm{P}$ bonds in molecules 4-7 (Figure 1c). For example, although ptBCZPO2TPTZ has the same $\mathrm{C}-\mathrm{P}$ bond as $\mathrm{CzPO} 2$ does, its $\mathrm{BDE}_{\mathrm{f}}(-)$ is as high as $3.15 \mathrm{eV}, 1 \mathrm{eV}$ higher than that of CzPO2. We found it did not show observable deterioration in laser-desorption/ionization time-of-flight mass spectrometry (LDI-TOFMS) until very high laser powers (Figure S1), suggesting its C-P bonds are relatively stable, although the authors did not consider the stability issues. ${ }^{[11 \mathrm{a}]}$ This finding indicates that the original fragile bonds can be turned into stable ones, which is rarely recognized in the previous development of OLED materials. It drove us to expound the underlying mechanism and explore a general effective strategy to manage $\operatorname{BDE}_{\mathrm{f}}(-)$, which will make an essential contribution to improving the operational stability of OLEDs and other organic (opto)electronic devices.

Herein, we first conducted comprehensive experiments and theoretical calculations, and revealed how $\operatorname{BDE}_{\mathrm{f}}(-)$ affects intrinsic material stability and device lifetime. Considering that the PO group is known to have $\operatorname{BDE}_{\mathrm{f}}(-)$ issues, but are still one of the most popular electron acceptors in high-efficiency hosts, ${ }^{[1]}$ PO-derivatives would be meaningful representatives for studying $\mathrm{BDE}_{\mathrm{f}}(-)$. In addition, $\mathrm{Cz}$ is nearly the most common electron donor in OLED molecules, ${ }^{[2]}$ and the corresponding $\mathrm{C}-\mathrm{N}$ bonds have demonstrated $\mathrm{BDE}_{\mathrm{f}}(-)$ issues in some cases, ${ }^{[6]}$ so $\mathrm{Cz}$ derivatives also deserve a thorough study. Thereby, we took a systematic theoretical study on typical PO and $\mathrm{Cz}$-derivatives, and developed an effective and general strategy to rationally manage $\operatorname{BDE}_{\mathrm{f}}(-)$ according to fundamental thermodynamics, which was further validated by the comparisons in several groups of reported and newly designed molecules. We uncovered that introducing an appropriate electron-withdrawing group (EWG) as the negative charge manager within the molecule can not only improve $\mathrm{BDE}_{\mathrm{f}}(-)$ substantially irrespective of the types of the fragile bonds (often by $\sim 1 \mathrm{eV}$ ), but also hinders the bond cleavage process greatly. This tactic provides a new perspective for reforming the originally vulnerable building blocks, thus largely enrich the alternative blocks for the development of robust OLED and other organic (opto)electronic materials.
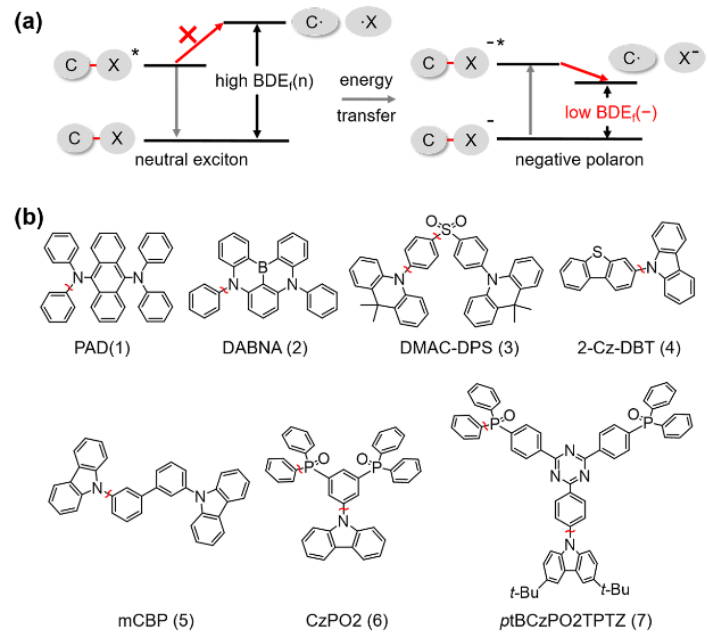

(c)

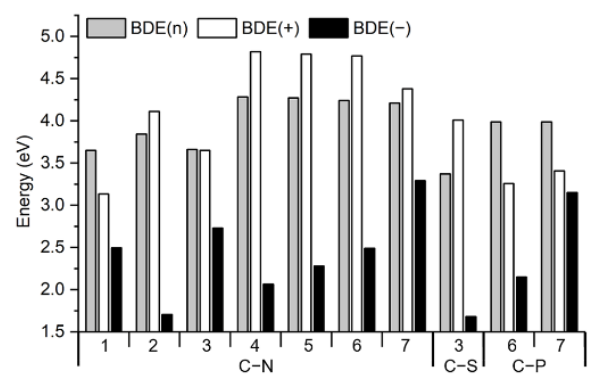

Figure 1. a) Schematic of the potential mechanism of EPA-induced bond-dissociation of the negative polaron. The asterisk refers to the excited state. BDEf(n) and $\mathrm{BDE}_{\mathrm{f}}(-)$ refers to the bond-dissociation energies of the fragile $\mathrm{C}-\mathrm{X}$ bonds under neutral and anionic states, respectively. b) Chemical structures of typical OLED molecules 1-7. The fragile bonds are highlighted by the red markers. $c) B D E_{f}$ values of the interested molecules. All calculations were at the M06-2X/def2-SVP level.

\section{Results and Discussion}

\section{Comparative Studies on TP3PO and PO-T2T}

To separate the concerned $\mathrm{BDE}_{\mathrm{f}}(-)$ from other material-related parameters (e.g. exciton energy and thermal stability), we comparatively studied the intrinsic stability of two representative PO-based electron-transporting materials (ETM), TP3PO and PO-T2T, which have 
very similar chemical structures (Figure 2a). ${ }^{[11 \mathrm{~d}, \mathrm{e}]}$ The structural similarity leads to many similar molecular parameters (Figure S2 and Table S1). As for molecular stability, $\mathrm{BDE}_{\mathrm{f}}(\mathrm{n})$ values in TP3PO and PO-T2T are all close to $4.00 \mathrm{eV}$ for the C-P bonds (more details are in Table S2), while their $\mathrm{BDE}_{\mathrm{f}}(-)$ values show large disparity, as low as 2.49 and $2.78 \mathrm{eV}$ for C1-P and C2-P bonds of TP3PO, but as high as 3.43 and $3.60 \mathrm{eV}$ for those of PO-T2T. The low $\mathrm{BDE}_{f}(-)$ of TP3PO would cause undesired chemical degradations, while the high $\mathrm{BDE}_{\mathrm{f}}(-)$ of PO-T2T is enough to afford exciton energies in most OLEDs, which may disburden this material of undesired degradations.

To validate these speculations, we conducted LDI-TOF-MS tests, which have proved to be powerful to study the chemical degradations of OLED materials. ${ }^{[5,6 \mathrm{c}-\mathrm{e}]}$ Here, samples were the pure powder of TP3PO and PO-T2T. Under the negative detection mode, the laser intensity was set to increase gradually from 90 to $110 \mu \mathrm{J} /$ pulse to track the degradation process (all spectra are in Figure S3). Even at the lowest intensity of $90 \mu \mathrm{J} /$ pulse, TP3PO showed weak molecular and quasi-molecular ion peaks ([M $\pm \mathrm{H}]^{-}$, etc.), and $\mathrm{C} 2-\mathrm{P}$ bond cleavage peaks $\left[\mathrm{M}-\mathrm{POPh}_{2}\right]^{-}$; but very strong $\mathrm{C} 1-\mathrm{P}$ bond cleavage peaks $[\mathrm{M}-\mathrm{Ph}]^{-}$and $[\mathrm{M}[\mathrm{O}]-\mathrm{Ph}]^{-}$. As the laser intensity increased, the $[\mathrm{M} \pm \mathrm{H}]^{-}$and $\left[\mathrm{M}-\mathrm{POPh}_{2}\right]^{-}$almost disappeared, while the $[\mathrm{M}-\mathrm{Ph}]^{-}$and $[\mathrm{M}[\mathrm{O}]-\mathrm{Ph}]^{-}$became very conspicuous. In comparison, at $90 \mu \mathrm{J} /$ pulse, $\mathrm{PO}-\mathrm{T} 2 \mathrm{~T}$ showed strong $\left[\mathrm{M} \pm \mathrm{H}^{-}\right.$, but very weak $[\mathrm{M}-\mathrm{Ph}]^{-}$and $[\mathrm{M}[\mathrm{O}]-\mathrm{Ph}]^{-}$. It is not until the intensity exceeded $100 \mu \mathrm{J} /$ pulse that the $[\mathrm{M}-\mathrm{Ph}]^{-}$and $[\mathrm{M}[\mathrm{O}]-\mathrm{Ph}]^{-}$became stronger than the $\left[\mathrm{M} \pm \mathrm{H}^{-}\right.$. The $\left[\mathrm{M}-\mathrm{POPh}_{2}\right]^{-}$of $\mathrm{PO}-\mathrm{T} 2 \mathrm{~T}$ was very weak throughout the entire process.

The LDI-TOF-MS results accord well with BDE predictions. Specifically, (i) For both TP3PO and PO-T2T, C1-P bonds with lower $\mathrm{BDE}$ are more fragile than $\mathrm{C} 2-\mathrm{P}$ bonds. (ii) TP3PO with lower $\mathrm{BDE}_{\mathrm{f}}(-)$ showed poorer material stability. These results strongly support that $\mathrm{BDE}_{f}$ is a key molecular parameter for the intrinsic material stability. Since QC-calculations and experiments both suggest that $\mathrm{C} 1-\mathrm{P}$ (C1 means the unsubstituted phenyl) are more fragile than $\mathrm{C} 2-\mathrm{P}$ bonds, the following studies on $\mathrm{PO}$-derivatives mainly focus on $\mathrm{C} 1-\mathrm{P}$ bonds.

\section{Device degradation Experiments}

We first fabricated TP3PO- and PO-T2T-based electron-only devices, with the structure ITO|PO-T2T (120 - $x \mathrm{~nm})|\mathrm{TP} 3 \mathrm{PO}(x \mathrm{~nm})| \mathrm{TPBi}$ $(20 \mathrm{~nm})|\mathrm{LiF}(1 \mathrm{~nm})| \mathrm{Al}(120 \mathrm{~nm})$ (Figure 2b). The current density-voltage curves of the devices are in Figure S4. Under the electrical stress $\left(10 \mathrm{~mA} \mathrm{~cm}^{-2}\right)$, we found that the aging of the EODs become more rapid as the thickness of TP3PO increases. For $x=0,5,20$, and 120 , the voltage increases $(\Delta V)$ of the EODs after $5 \mathrm{hr}$ stress are $0.04,1.57,4.41$, and $20.52 \mathrm{~V}$, respectively (Figure $2 \mathrm{c}$ ). This result clearly demonstrates that the instability of TP3PO anion is the main cause of the voltage rise. Next, we fabricated OLEDs with the structure ITO|HATCN $(10 \mathrm{~nm})|\mathrm{NPB}(30 \mathrm{~nm})|$ TCTA $(15 \mathrm{~nm})|\mathrm{mCBP}(15 \mathrm{~nm})| \mathrm{DPEPO}: 30 \mathrm{wt} \%$ TCzTrz $(30 \mathrm{~nm}) \mid \mathrm{PO}-\mathrm{T} 2 \mathrm{~T}(40-x \mathrm{~nm})$ |TP3PO $(x \mathrm{~nm})|\operatorname{TPBi}(5 \mathrm{~nm})| \mathrm{LiF}(1 \mathrm{~nm}) \mid \mathrm{Al}(120 \mathrm{~nm})(x=5,10,30$, or 35; Figure $2 \mathrm{~d})$. Chemical structures of the involved organic materials are shown in Figure S5. TCzTrz is a sky-blue emitter developed by Zhang et al. ${ }^{[12]}$ All these OLEDs demonstrated close maximum efficiencies $(\sim 11 \%$, Figure S5), which are comparable to reported values. Figure $2 e$ shows their half-lifetime (LT50) at an initial brightness of $500 \mathrm{~cd} \mathrm{~m}^{-2}$. For the devices $x=5,10,30$, and 35, LT50 values are 12.0, 11.0, 7.7, and 4.2 hours, respectively (Figure 2e). Since the only difference between those devices is the thicknesses of TP3PO and PO-T2T, the result further supports that it is the TP3PO with much lower $\operatorname{BDE}_{\mathrm{f}}(-)$ that mainly accounts for device degradations.

To rationally link the macroscopic device degradation with the microscopic bond cleavage, we further calculated energy levels of POcontaining anions resulted from the bond cleavage of TP3PO and PO-T2T. Figure S6 shows that HOMOs of PO-containing anions $(-1.72$ to $-2.20 \mathrm{eV})$ are much lower than LUMOs $(-0.79$ to $-1.27 \mathrm{eV})$ of the intact molecules. Hence, once generated, those anions would act as filled deep traps, hindering the electron transport and injection. Since TP3PO is more fragile towards electrons, the corresponding devices will generate much more defects in the same time scale, thus leading to bigger voltage increase and shorter device lifetime.

Up to here, we comprehensively demonstrated the close relationship between $\operatorname{BDE}_{f}(-)$, intrinsic material stability, and device lifetime, and revealed that active organic materials with lower $\mathrm{BDE}_{\mathrm{f}}(-)$ would result in poorer material stability and device lifetime. It is noteworthy that the comparison between TP3PO and PO-T2T demonstrates that with appropriate molecular design, the originally vulnerable groups like $\mathrm{PO}$ can serve in robust materials, although that is rarely recognized before. Therefore, it would be imminent to reveal the relationship between $\operatorname{BDE}_{\mathrm{f}}(-)$ and molecular structure, and establish feasible strategies to improve $\operatorname{BDE}_{\mathrm{f}}(-)$. 
(a)
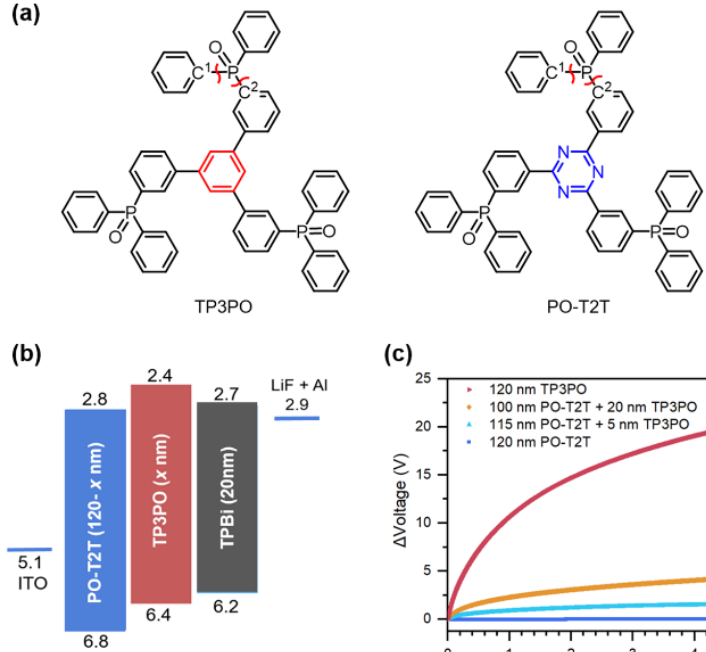

(c)

(d)

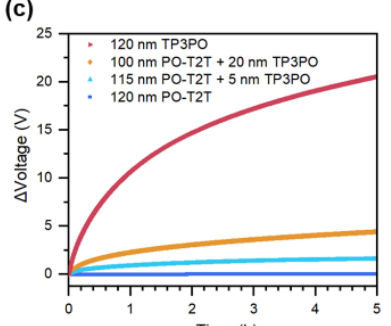

(e)
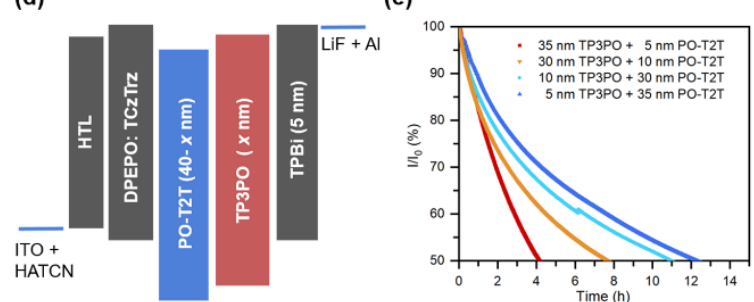

Figure 2. a) Chemical structures of TP3PO and PO-T2T, the fragile bonds are highlighted by the red markers. b) Device structures of the EODs $(x=0,5,20$, or 120). c) Change of the voltages of EODs during 5 hours under a constant current density of $10 \mathrm{~mA} \mathrm{~cm}^{-2}$. d) Device structures of the OLEDs ( $x=5$, 10, 30, or 35). To prevent the introduction of new interfaces, devices with solely TP3PO (or PO-T2T) as the ETM were not fabricated. e) The operation lifetime of the OLEDs measured at a brightness of $500 \mathrm{~cd} \mathrm{~m}^{-2}$ under a constant current.

\section{Key Influence Factors of $\operatorname{BDE}_{\mathrm{f}}(-)$}

For the $\mathrm{C}-\mathrm{X}(\mathrm{X}=\mathrm{N}, \mathrm{P}, \mathrm{S}$, etc.) bonds in OLED molecules, $\mathrm{X}$-sides usually have higher electronegativities, so the bond dissociations in anionic states will result in anions containing $X$. According to the Hess's law, an equation between $\operatorname{BDE}_{f}(-)$ and $\operatorname{BDE}_{f}(n) \operatorname{can}_{\text {be derived }}$ (Scheme 1)

$$
\operatorname{BDE}_{\mathrm{f}}(-)=\operatorname{BDE}_{\mathrm{f}}(\mathrm{n})+\mathrm{EA}_{\mathrm{m}}-\mathrm{EAx}
$$

Scheme 1. Derivation of the calculation formula of $\operatorname{BDE}_{\mathfrak{f}}(-)$.

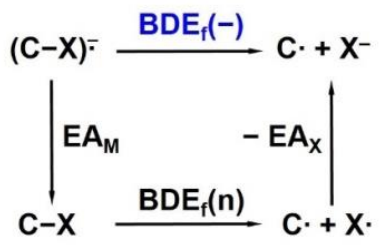

where $E A_{X}$ and $E A_{M}$ represent the electron affinity of the $X$ radical and the intact molecule, respectively. For all $C-X$ bonds in Figure $1 b$, it is their $E A_{X}$ values $(2-2.6 \mathrm{eV})$ significantly higher than $E A_{M}$ values $(0.3-1.5 \mathrm{eV})$ that lead to largely reduced $B D E_{f}(-)$ by over 1 $\mathrm{eV}$. Most importantly, equation (1) suggests how to regulate $\operatorname{BDE}_{f}(-)$. For the first term $\operatorname{BDE}_{f}(n)$, it mainly depends on the type of $C-X$ bond and rarely influenced by other parts of the molecule except ortho-substituents. ${ }^{\left[{ }^{[i, 6 j]}\right]}$ Although ortho-substituents may cause large influences (up to $0.5 \mathrm{eV}$ ), the effects depend on case-specific spatial factors and electronic characters of the substituents. So the orthoeffects are not involved here. For $E A_{M}$, it can be ideally increased by introducing $E W G$ on $C$-side of $C-X$ bond. It is feasible when $X$ is an atom (e.g. halogen). However, in OLED molecules, X-sides are usually building blocks. Moreover, many OLED molecules have several $\mathrm{C}-\mathrm{X}$ bonds (Figure $1 \mathrm{~b}$ ), modifications on the $\mathrm{C}$-side of one $\mathrm{C}-\mathrm{X}$ bond may be right on the $\mathrm{X}$-sides of the others. In these cases, substitutions may lead to a nearly equivalent increase of $E A_{M}$ and $E A_{X}$, resulting in a limited increase or even decrease of $B D E_{f}(-)$.

Based on the foregoing discussion, to obtain high $\operatorname{BDE}_{f}(-)$ for a given $C-X$ bond, an appropriate EWG is required to manage the negative charge, called negative charge manager. For that purpose, it should meet three primary criteria: First, it should be sufficietly stable without any fragile bonds in itself; second, it must be able to increase $E A_{M}$ greatly; third, it must be able to control the increase of $E A_{x}$. So in the next, we screened for the suitable $E W G$ as negative charge managers to improve $B D E_{f}(-)$ of various fragile bonds for robust OLED molecules. 


\section{Improving $\mathrm{BDE}_{\mathfrak{f}(-)}$ Values of PO-derivatives}

We first took $\mathrm{POPh}_{3}(\mathbf{P 1})$ as the parent molecule. Figure 3a shows the negative charge in $\mathrm{POPh}_{3}$ anion mainly distributes on its delocalized LUMO, while that of the $\mathrm{POPh}_{2}$ fragment anion mainly locates on $\mathrm{sp}^{3}$-orbital of $\mathrm{P}$. Thus, EA $\mathrm{A}_{M}$ and $E A_{x}$ would be basically determined by the distinct orbitals. To increase $E A_{M}$, introducing strong EWG or delocalizing structures are two typical ways. Meanwhile, they would have smaller impact on EAx due to the localization of the $\mathrm{sp}^{3}$-orbital. Accordingly, we designed P2-P4 with stable and strong EWGs, including trifluoromethyl (P2), cyano (P3), or replace one of the phenyl with 1,3,5-triazine (P4); and P5-P6 with relatively weaker electron-withdrawing character but delocalizing structures, including pyridine (P5) and [1,1':3',1"-terphenyl]-5'-yl (PTP) (P6) (Figure 3b). Calculations show that $E A_{M}$ values of P2-P6 all increase by $0.6-1 \mathrm{eV}$, higher than the increase of $E A_{x}$ values $(\leq 0.45 \mathrm{eV})$. Of note, $E A_{x}$ values of P5-P6 are $\sim 0.2 \mathrm{eV}$ smaller than those of P2-P4. In total, $\mathrm{BDE}_{\mathrm{f}}(-)$ values of P2-P6 increase to 2.15-2.55 eV, which are considerably higher than that of $\mathbf{P 1}(1.87 \mathrm{eV})$, yet still lower or comparable to the $\mathrm{BDE}_{\mathrm{f}}(-)$ of the unstable TP3PO $(2.49 \mathrm{eV})$. Thus, the substituents above do not satisfy the second criteria well, a greater enhancement of $E A_{M}$ is demanded. Incidentally, the same substituents at meta-positions of $\mathrm{P}$ atom bring similar effects (Table S3).

Since strong EWG and delocalizing structures can both increase $E A_{M}$, and the latter has a smaller influence on $E A x$, we thus combined the two methods, namely introducing delocalized strong EWG (D-EWG) as the negative charge manager to further improve $\mathrm{BDE}_{\mathrm{f}}(-)$. P7 and P8 were designed accordingly. Indeed, introductions of benzonitrile in P7 and 4,6-diphenyl-1,3,5-triazin-2-yl (Trz) in P8 remarkably increase $E A_{M}$ by over $1.2 \mathrm{eV}$. Moreover, they could suppress the increase of EAx. For instance, P3 and P7 both have a cyano; while $\mathbf{P 7}$ has an increased distance between the cyano and $\mathrm{P}$ atom, leading to a smaller $E A_{x}$ increase $(0.32$ vs $0.45 \mathrm{eV}$ for $\mathbf{P 7}$ and P3). Meanwhile, neither benzonitrile or Trz have fragile $C-X$ bonds, so these D-EWGs meet all the required conditions above. As anticipated, they significantly improves $\mathrm{BDE}_{\mathrm{f}}(-)$ to 2.73 and $3.04 \mathrm{eV}$ for $\mathbf{P 7}$ and P8 (Figure 3b), much higher than that of the unstable TP3PO. Therefore, introducing D-EWG as the negative charge manager could be an effective strategy for improving $\mathrm{BDE}_{\mathrm{f}}(-)$ greatly, which does outperform the effects of solely introducing of strong EWGs or delocalizing structures in P2-P6. For aryl amide and sulfone derivatives, these managers likewise increase their $\operatorname{BDE}_{\mathrm{f}}(-)$ values by $\sim 1 \mathrm{eV}$ (Table S4).

(a)

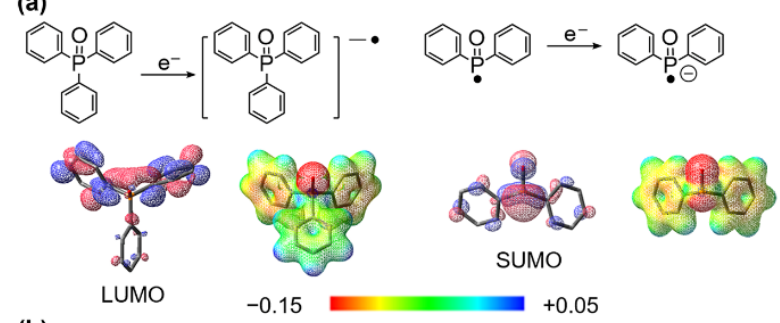

(b)

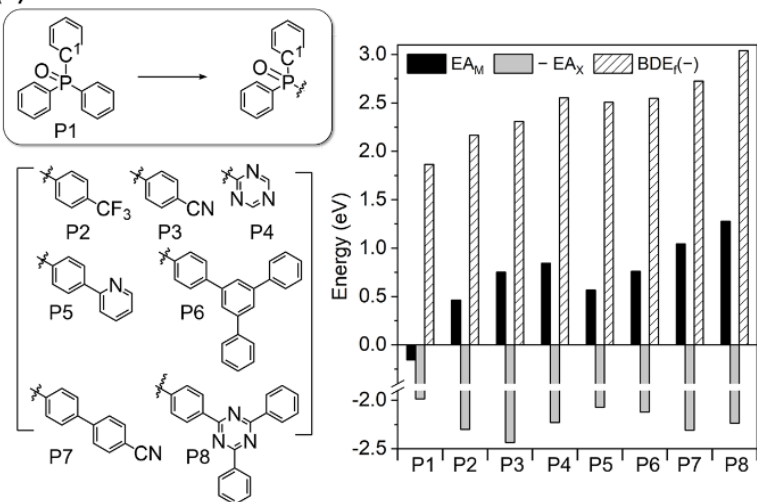

Figure 3. a) Frontier orbital (isovalue $=0.03 \mathrm{au}$ ) and $\mathrm{ESP}$ maps of $P O P h_{3}$ and $P O P h_{2}$. b) Chemical structures, $E A_{M}, E A x$, and $B D E_{f}(-)$ values $($ with respect to $C 1-P$ bond) of molecules P1-P8. All calculations were at the M06-2X/def2-SVP level.

Notably, the aforementioned TP3PO and PO-T2T involve P6 and P8 as substructures, respectively; so their stability difference can be likewise rationalized. Compared with TP3PO, PO-T2T with a D-EWG has a significantly improved EA $(1.58 \mathrm{vs} 0.77 \mathrm{eV}$ ), but a slightly decreased $E A_{x}(2.07$ vs $2.22 \mathrm{eV})$, thus leading to $~ 1 \mathrm{eV}$ improvment for $\mathrm{BDE}_{\mathrm{f}}(-)$. That further supported that $\mathrm{D}$-EWGs meet the criterias of negative charge mangers well. In addition, $E A_{M}$ of PO-T2T is $0.3 \mathrm{eV}$ higher than that of P8 (1.58 vs $1.28 \mathrm{eV}$ ), demonstrating that the D-EWG Trz at the center of the molecule makes all the PO groups interconnected to further promote $E A_{M}$, leading to much higher $\mathrm{BDE}_{\mathrm{f}}(-)$ of PO-T2T (3.43 vs $\left.3.04 \mathrm{eV}\right)$. Moreover, the negative charge managers not only improve the thermodynamic stability, as reflected by $\mathrm{BDE}_{\mathrm{f}}$, but suppress the kinetic process of bond cleavages. That is confirmed by the comparison between the rapid degradation of TP3PO-only EOD, and the robustness of PO-T2T-only EOD. During C-P bond cleavages towards electrons, the electron on LUMO redistributes to $\mathrm{sp}^{3}$-orbital of $\mathrm{P}$. When there is a negative charge manager, the redistribution will become more difficult. To visualize this, potential energy curves (PECs) and electrostatic potential (ESP) maps are plotted during C1-P bond cleavages in TP3PO and PO-T2T anions. Figure 4a shows that PECs of the two anions are almost identical when C-P distance 
is $\sim 1.8 \AA$. However, when the distance is over $2.6 \AA$, PECs of PO-T2T clearly increase faster than those of TP3PO. In ESP maps (Figure $4 \mathrm{~b}$ ), when C1-P distance is $1.8 \AA$, the negative ESP (in red) is mainly allocated around the center for both molecules (also see Figure S7). While at $2.6 \AA$, the red color in the central TP3PO becomes shallower; while that in PO-T2T scarcely changes. From $1.8 \AA$ to $2.6 \AA$, the negative charge allocated around the central aryl $\left(q_{\mathrm{Ar}}\right)$ changes from -0.76 to -0.60 for TP3PO, while in PO-T2T, $q_{\mathrm{Ar}}$ only changes from -0.75 to -0.72 . Finally, when the phenyl leaves, the negative charge is concentrated on PO, while $q_{\mathrm{Ar}}$ of PO-T2T is still higher than that of TP3PO ( -0.35 vs -0.27 , Table S5). This result clearly reflects that Trz as negative charge manager can effectively confine the negative charge within itself, thus protect $\mathrm{C}-\mathrm{X}$ bonds and improve their kinetic stability towards electrons. Next, we further demonstrate that the strategy is also effective for $\mathrm{Cz}$-derivatives, even though $\mathrm{Cz}$ and $\mathrm{PO}$ have quite different electronic structures.

(a)

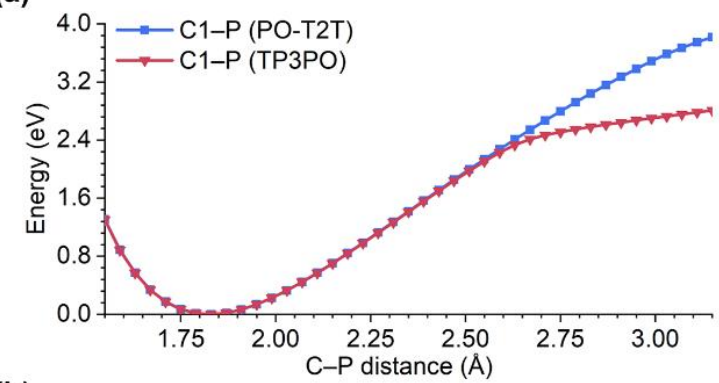

(b)

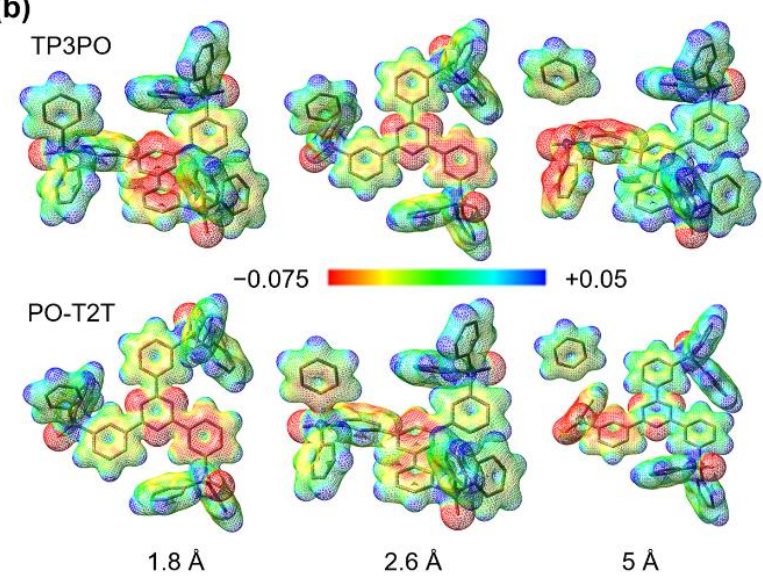

Figure 4. a) Potential energy curves with respect to the stretch of C1-P bonds $(1.55-3.15 \AA$ ) in TP3PO and PO-T2T anions. b) ESP maps (isovalue $=0.02$ au) when the C1-P distance is $1.8,2.6$, and $5 \AA$, respectively. All calculations were at the M06-2X/def2-SVP level.

\section{Improving $\mathrm{BDE}_{\mathrm{f}}(-)$ Values of $\mathrm{Cz}$-derivatives}

For $\mathrm{Cz}$-derivatives, substituents on $\mathrm{N}$-side or $\mathrm{C}$-side of the $\mathrm{C}-\mathrm{N}$ bond would have distinct effects on $E A_{M}$ and $E A_{X}$. We first studied the substituent effects on $\mathrm{N}$-side. Based on the parent PhCz (C1), we introduced the cyano, phenyl PTP, and phenyl Trz groups into 2 and 3-sites of $\mathrm{Cz}$, respectively. Defferent from $\mathrm{POPh}_{2}$ fragment anion with localized $\mathrm{SUMO}$ on $\mathrm{sp}^{3}$-orbital of $\mathrm{P}, \mathrm{Cz}$ radical has delocalized SUMO. ${ }^{[6]]}$ So it would be more difficult to control the increase of EAx to meet the third criteria. Indeed, at 3-site, strong EWG cyano considerably increases EAx by $0.63 \mathrm{eV}$ due to its large contribution to the SUMO (Figure S8). Such increase is even higher than that of $\mathrm{EA}_{\mathrm{M}}(0.5 \mathrm{eV}$, Figure $5 \mathrm{~b})$, resulting in a decreased $\mathrm{BDE}_{\mathrm{f}}(-)$ (1.39 vs $1.45 \mathrm{eV}$ for $\mathbf{C 2}$ and $\left.\mathbf{C} 1\right)$. In comparison, PTP with delocalizing structrure brings much smaller increase of $E A_{X}(0.25 \mathrm{eV})$, but bigger increase of $E A_{M}(0.7 \mathrm{eV})$, thus leading to an increased $B D E_{f}(-)$ $(1.89 \mathrm{eV})$ for C3. Likewise, the D-EWG Trz brings a smaller increase of $E A_{x}(0.36 \mathrm{eV})$, but a much bigger increase of $E A_{M}(1.36 \mathrm{eV})$, thus leading to a significantly increased $\mathrm{BDE}_{\mathrm{f}}(-)(2.42 \mathrm{eV})$ for $\mathbf{C 4}$, about $1 \mathrm{eV}$ higher than that of $\mathbf{C}$. Therefore, the D-EWG Trz still meets all the three criteria, serving well as a negative charge manager.

Intriguingly, $\mathrm{BDE}_{\mathrm{f}}(-)$ values of the 2-substituted isomers are 1.78, 2.15, and $2.62 \mathrm{eV}$ for $\mathbf{C 5}$, $\mathbf{C 6}$, and $\mathbf{C 7}$, respectively; which are 0.2-0.4 eV higher than those of 3-substituted isomers. That is because the substituent at 2-site has a stronger interaction with the LUMO of Cz, but a relatively weaker interaction with the SUMO of $\mathrm{Cz}$ radical (Figure S9). Although 3,6-substitutions are widely used in Cz-derivatives, the result here shows that 2,7-substitutions has greater advantage on improving the intrinsic molecular stability. In principle, substitutions on $\mathrm{C}$-side of $\mathrm{PhCz}$-derivatives solely increase $\mathrm{EA}_{\mathrm{M}}$ and thus improve $\mathrm{BDE}_{\mathrm{f}}(-)$. As shown in Figure $5 \mathrm{~b}$, the cyano and $\mathrm{Trz}$ on the $\mathbf{C}$-side of $\mathbf{C 8}$ and $\mathbf{C 9}$ significantly increases their $\mathrm{BDE}_{\mathrm{f}}(-)$ to 2.26 and $3.02 \mathrm{eV}$, which are much higher than those of the $\mathrm{N}$-side substituted counterparts. 

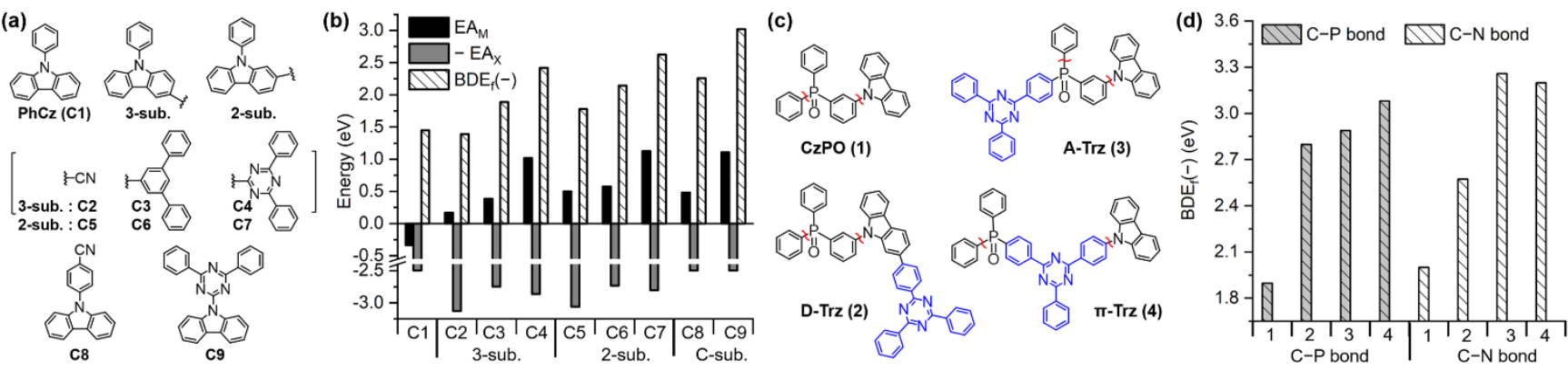

Figure 5. a) Chemical structures of $\mathrm{PhCz}$ and its derivatives, $B D E_{f}(-)$ values of the fragile $\mathrm{C}-\mathrm{N}$ bonds were calculated. b) $E A_{M}, E A x$, and $B D E_{f}(-)$ values of $P h C z$ derivatives $\mathrm{C} 1-\mathrm{C}$. c) Chemical structures of CzPO-derivatives with $\mathrm{D}-\pi-\mathrm{A}$ structures. The fragile $\mathrm{C}-\mathrm{N}$ and $\mathrm{C}-\mathrm{P}$ bonds are highlighted by the red markers. d) $\mathrm{BDE}_{\mathrm{f}}(-)$ values of $\mathrm{CzPO}$-derivatives. All calculations were at the M06-2X/def2-SVP level.

\section{Improving $\operatorname{BDE}_{\mathfrak{f}}(-)$ Values of Donor (D)- - -Acceptor $(A)$ Molecules}

Most OLED molecules contain D-ח-A structure, in which the fragile bonds are either in $D, A$ or in both. To explore the distinct effects of substituted position in D- $\Pi-A$ molecules, we designed a group of molecules based on (3-(9H-carbazol-9yl)phenyl)diphenylphosphineoxide ( $\mathrm{CzPO}$, Figure $5 \mathrm{c}$ ), with $\mathrm{Cz}$ and $\mathrm{PO}$ as $\mathrm{D}$ and $\mathrm{A}$, respectively. Its $\mathrm{C}-\mathrm{P}$ and $\mathrm{C}-\mathrm{N}$ bonds have comparable $\mathrm{BDE}(-)$ values $(1.90 \mathrm{vs} 2.00 \mathrm{eV})$. Trz, as an effetive negative charge manager, is introduced to the 2-site of $\mathrm{Cz}$ (D-Trz),

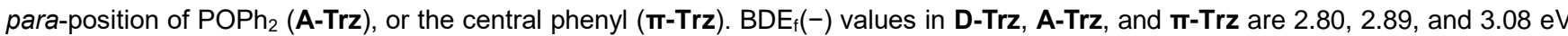
for $\mathrm{C}-\mathrm{P}$ bonds; and 2.67, 3.26, and $3.20 \mathrm{eV}$ for $\mathrm{C}-\mathrm{N}$ bonds, respectively (Figure $5 \mathrm{~d}$ ). These values are $0.67-1.26 \mathrm{eV}$ higher than those of CzPO. Compared with D- or A-substituted counterparts, $\pi$-Trz simultaneously reinforces all the fragile bonds from $D$ and $A$. The framework not only makes each group contribute to $E A_{M}$, but also keeps them being spatially separated, preventing them from increasing $E A_{x}$ (the details are in Figure $\mathrm{S10}$ ). Therefore, employing negative charge managers at the $\pi$-bridge of the molecule is most recommended for developing robust $\mathrm{D}-\pi-\mathrm{A}$ molecules. It has been well evidenced by the comparison of CzPO2 and $p \mathrm{tBCZPO} 2 \mathrm{TPTZ}$. The D-EWG Trz at the center of the ptBCZPO2TPTZ serves well as an effective negative-charge manager, thus improves both $\mathrm{C}-\mathrm{N}$ and $\mathrm{C}-\mathrm{P}$ bonds by $\sim 1 \mathrm{eV}$ in comparison to those of $\mathrm{CzPO} 2$.

\section{Implications for Rational Design of Robust OLED Materials}

In OLED molecules, many building blocks can contribute to desirable optical/electrical performance. PO is a representative instance, which often plays an important role in the corresponding high-efficiency materials. ${ }^{[1]}$ Unfortunately, when PO is the sole acceptor, the corresponding molecules suffer from low $\mathrm{BDE}_{\mathrm{f}}(-)$ values and poor material stability in most cases, as clearly demonstrated by $\mathrm{CzPO} 2$ and TP3PO. Similar issues would occur in A1-A3 as evidenced by the low BDE $\mathrm{F}_{\mathrm{f}}(-)$ values $(1.48-2.33 \mathrm{eV}$, Figure $6 \mathrm{a}$ and $6 \mathrm{~b})$. To enhance the molecular stability towards electrons, exclusively using strong and stable acceptors is indeed viable. The Trz, cyano, and carbonyl undoubtedly guarantee high $\mathrm{BDE}_{\mathrm{f}}(-)$ values (2.81-3.33 eV for B4-B6, Figure 6c). Nevertheless, abundant building blocks are always desired to meet the various needs in practical applications.

Here, we demonstrated a superior strategy by introducing or constructing an appropriate D-EWG as the negative charge manager to make fragile bonds no longer fragile towards electrons. The effectiveness can be evidenced by $\mathbf{C 1 - C 4}$, which retain the building blocks and fragile bonds in A1-A4, but combine them with an ancillary strong EWGs or delocalizing structures, constructing an effective negative charge manager within the molecule. Markedly different from A1-A4, C1-C4 have substantially improved $B D E_{f}(-)$ values (2.75-3.17 eV, Figure 6b). Meanwhile, those groups like PO could bring desirable optical and electrical performance, as evidenced by C4 with a tenfold increase of radiation rate constant and a fivefold decrease of non-radiation rate constants compared with PO-free counterparts. ${ }^{[11 a]}$

Our study for the first time disclosed the strategy can not only preserve the intrinsic advantages of the building blocks, but also address the intrinsic stability issues. The improved molecular stability of C1-C4 can be evidenced by the experiments. Lee et al. found that $\mathbf{C 1}$-based EOD are robust towards electrons. ${ }^{[13]}$ Kwon et al. replaced $\mathbf{A} 1$ with $\mathbf{C} 1$ as the host of a blue-OLED, which gave a $\sim 7$ fold increase of device lifetime. ${ }^{[\mathrm{k}]}$ Duan et al. found the C2-based OLEDs have a substantially prolonged lifetime compared with that based on $\mathbf{A 2}{ }^{\left[{ }^{14]}\right]}$ in which the significantly increased $\mathrm{BDE}_{\mathrm{f}}(-)$ should not be neglected. $\mathbf{C} \mathbf{3}$ is reported as a robust host even for pure blue phosphorescent OLEDs. ${ }^{[15]}$ The superior molecular stability of C4 to A4 has been demonstrated above, although the authors did not mentioned this point.

To enrich the building blocks for robust OLED materials, we designed three new blocks D1-D3 (Figure 6a) as negative charge managers. We expect they can stabilize various fragile bonds from electrons, and thus allowing high tunability for molecular design. Indeed, whether combined with $\mathrm{PO}$ or $\mathrm{Cz}$, the corresponding molecules all show high $\mathrm{BDE}_{\mathrm{f}}(-)$ values $(2.87-3.55 \mathrm{eV}$, Figure $6 \mathrm{~d})$. Finally, we show that the strategy is also effective for systems other than $\mathrm{Cz} / \mathrm{PO}$. For ETMs based on benzimidazole-derivatives, the prototypical TPBi (E1) has particularly low $\mathrm{BDE}_{\mathrm{f}}(-)(1.61 \mathrm{eV})$ for the $\mathrm{C}-\mathrm{N}$ bond. In comparison, E2 with extended m-conjugation has a higher $\mathrm{BDE}_{\mathrm{f}}(-)$ $(2.54 \mathrm{eV})$. Indeed, E2-based OLEDs have a 3 fold increase of device lifetime compared with E1-based counterparts, ${ }^{[16]}$ in which the improved $\mathrm{BDE}_{\mathrm{f}}(-)$ should have a non-negligible contribution. Based on E2, we constructed 9,10-di(pyrimidin-2-yl)anthracene as a negative charge manager, which further improves $\operatorname{BDE}_{\mathrm{f}}(-)$ for $\mathbf{E 3}(2.92 \mathrm{eV}$, Figure 6e). It is certain that the newly designed E3 with substantially higher $\operatorname{BDE}_{\mathrm{f}}(-)$ would be robust towards electrons. Overall, these examples further consolidate that our strategy would be 
general and effective for improving the molecular stability of various OLED materials towards electrons.
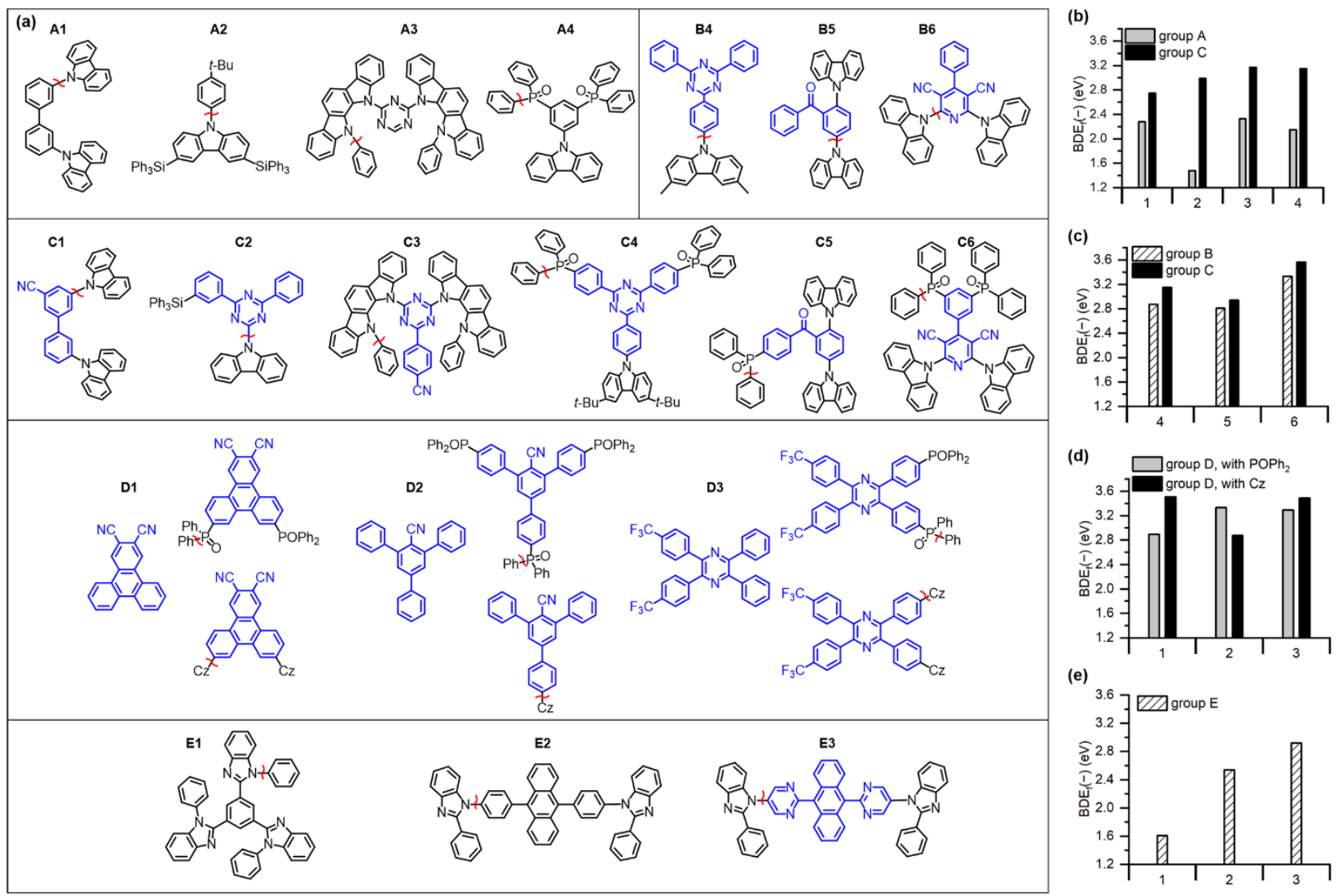

(e)

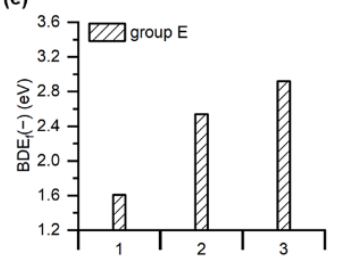

Figure 6. a) Chemical structures of the reported or newly designed OLED molecules. Their fragile bonds are highlighted by the red markers. $b-e), B D E_{f}(-)$ values of the molecules in: group $A$ and $(\mathrm{b}$ ); group $\mathrm{B}$ and $\mathrm{C}$ c); group D d); and group $\mathrm{E}$ e). Among which, molecule $\mathrm{C} 5, \mathrm{C} 6, \mathrm{E} 3$, and all of the molecules in group D are newly designed in this work. The negative charge managers are highlighted in blue colour. All calculations were at the M06-2X/def2-SVP level.

\section{Conclusion}

In this work, we first emphasized $\mathrm{BDE}_{\mathrm{f}}(-)$ is of vital importance but less-studied molecular parameter for OLED materials, and confirmed the close relationship between $\operatorname{BDE}_{f}(-)$, material stability, and device lifetime. The comparative study on typical ETMs TP3PO and PO-T2T clearly demonstrated that active organic materials with lower $\mathrm{BDE}_{\mathrm{f}}(-)$ do result in poorer material stability and device lifetime. We then explored how to manipulate $\mathrm{BDE}_{f}(-)$. Based on the fundamental Hess's law, regulating $\mathrm{BDE}_{\mathrm{f}}(-)$ is converted to the explicit modulations of $E A_{M}$ and $E A_{x}$. We found that introducing appropriate delocalized strong $E W G$ as negative charge manager can significantly improve $E A_{M}$, but has much smaller effect on $E A_{x}$, thus substantially improving the $\operatorname{BDE}_{f}(-)$ by $\sim 1$ eV for various OLED molecules. Meanwhile, such managers can well confined the negative charge in themselves and thus hinder the bond cleavage process greatly. In particular for the D-ח-A molecules with multiple fragile bonds, the introduction of the manager at the $\pi$-bridge of the molecule is most recommended, which can stabilze all the fragile bonds. The effectiveness and generality of this strategy has been further validated in several newly designed molecules. Importantly, it provides a particular perspective to reform the originally vulnerable building blocks no longer vulnerable towards electrons, thus largely enriches available building blocks for the rational design of robust OLED materials. At the end, it should be noted that this research looked into the regulations of $\operatorname{BDE}(-)$ of $C-X$ bonds, which helps deepen the fundemental understanding on the relationship between molecular structures and BDE(-) values for all organic compounds. Hence, the strategy would be transferrable to other organic (opto)electronic materials.

\section{Acknowledgements}


This work was supported by the National Key R\&D Program of China (No. 2016YFB0401003, 2016YFB0400702) and the National Science Fund of China (Grant Nos. 51525304) for financial support. We are grateful to Prof. Hui Xu et al. and Prof. Qisheng Zhang et al. for providing the sample of ptBCzPO2TPTZ and TCzTrz, respectively. We are also grateful to Prof. Jinpei Cheng and Dr. Jindong Yang for their valuable suggestions on our manuscript. We thank the Tsinghua National Laboratory for Information Science and Technology and Tsinghua Xuetang Talents Program for supporting the computational resources.

Keywords: anions $\bullet$ bond energy $\bullet$ chemical deterioration $\bullet$ intrinsic stability $\bullet$ organic light-emitting diodes

[1] a) O. Ostroverkhova, Chem. Rev. 2016, 116, 13279-13412; b) W. R. Mateker, M. D. McGee, Adv. Mater. 2017, 29, 1603940; c) J. T. E. Quinn, J. Zhu, X. Li, J. Wang, Y. Li, J. Mater. Chem. C, 2017, 5, 8654-8681. d) S. Scholz, D. Kondakov, B. Lüssem, K. Leo, Chem. Rev. 2015, 115, 8449-8503.

[2] a) C. Zhao, L., Duan, J. Mater. Chem. C, 2020, 8, 803-820; b) W. Song, J. Y. Lee, Adv. Opt. Mater. 2017, 5, 1600901; c) S. Schmidbauer, A. Hohenleutner, B. König, Adv. Mater. 2013, 25, 2114-2129; d) S. K. Jeon, H. L. Lee, K. S., Yook, J. Y. Lee, Adv. Mater. 2019, 31, 1803524; e) X. Cai, S.-J. Su, Adv. Funct. Mater. 2018, 28, 1802558.

[3] a) N. C. Giebink, B. W. D’Andrade, M. S. Weaver, P. B. Mackenzie, J. J. Brown, M. E. Thompson, S. R. Forrest, J. Appl. Phys. 2008, 103, No. 044509; b) M. Shao, L. Yan, M. Li, I. Ilia, B. Hu, J. Mater. Chem. C 2013, 1, 1330-1336; c) Y. Zhang, J. Lee, S. R. Forrest, Nat. Commun. 2014, 5, 5008; d) W. Song, J. Y. Lee, T. Kim, Y. Lee, H. Jeong, Org. Electro. 2018, 57, 158-164; e) J. Sohn, D. Ko, H. Lee, J. Han, S.-D. Lee, C. Lee, Org. Electron. 2019, 71, 286-291; f) H. Xu, M. Wang, Z.-G. Yu, K. Wang, B. Hu, Adv. Phys. 2019, 68, 49-121; g) M. Tanaka, R. Nagata, H. Nakanotani, C. Adachi, Commun. Mater. 2020 , 1:18.

[4] a) D. Zhang, M. Cai, Y. Zhang, D. Zhang, L. Duan, Mater. Horiz. 2016, 3, 145-151; b) J. Lee, C. Jeong, T. Batagoda, C. Coburn, M. E. Thompson, S. R. Forrest, Nat. Common. 2017, 8:15566; c) H. Noda, H. Nakanotani, C. Adachi, Sci. Adv. 2018, 4: eaao6910; d) A. Ligthart, X. de Vruesm, L. Zhang, M. C. W. M. Pols, P. A. Bobbert, H. van Eersel, R. Coehoorn, Adv. Funct. Mater. 2018, 1804618; e) J. S. Bangsund, K. W. Hershey, J. R. Holmes, ACS Appl. Mater. Interfaces 2018, 10, 5693-5699; f) D.-G. Ha, J. Tiepelt, M. A. Fusella, M. S. Weaver, J. J. Brown, M. Einzinger, M. C. Sherrott, T. V. Voorhis, N. J. Thompson, M. A. Baldo, Adv. Optical Mater. 2019, 1901048; g) S. H. Han, J. H. Jeong, J. W. Yoo, J. Y. Lee, J. Mater. Chem. C, $2019,7,3082-3089$.

[5] C. Jeong, C. Coburn, M. Idris, Y. Li, P. I. Djurovich, M. E. Thompson, S. R. Forrest, Org. Electron. 2019, 64, 15-21;

[6] a) D. Y. Kondakov, W. C. Lenhart, W. F. Nichols, J. Appl. Phys. 2007, 101, No. 024512; b) D. Y. Kondakov, J. Appl. Phys. 2008, 104, No. 084520; c) S. Scholz, K. Walzer, K. Leo, Adv. Funct. Mater. 2008, 18, 2541-2547; d) N. Lin, J. Qiao, L. Duan, H., Li, L. Wang, Y. Qiu, J. Phys. Chem. C 2012, 116, 1945119457; e) N. Lin, J. Qiao, L. Duan, L. Wang, Y. Qiu, J. Phys. Chem. C 2014, 118, 7569-7578; f) A. S. D. Sandanayaka, T. Matsushima, C. Adachi, J. Phys. Chem. C 2015, 119, 23845-23851; g) S. Y. Byeon, S. H. Han, J. Y. Lee, Adv. Optical Mater. 2017, 5, 1700387; h) A. Y. Freidzon, A. A. Safonov, A. A. Bagaturyants, D. N. Krasikov, B. V. Potapkin, A. A. Osipov, A. V. Yakubovich, O. Kwon, J. Phys. Chem. C 2017, 121, 22422-22433; i) R. Wang, Y.-L. Wang, N. Lin, R. Zhang, L. Duan, J. Qiao, Chem. Mater. 2018, 30, 8771-8781; j) M. Hong, M. K. Ravva, P. Winget, J.-L. Brédas, Chem. Mater. 2016, 28, 57915798. k) D. H. Ahn, J. H. Maneg, H. Lee, H. Yoo, R. Lampande, J. Y. Lee, J. H. Kwon, Adv. Optical Mater. $2020,8,2000102$.

[7] Y. Luo, H., Aziz, G., Xu, Z. D. Popovic, Chem. Mater. 2007, 19, 2079-2083

[8] T. A. Schaub, E. M. Zolnhofer, D. P. Halter, T. E. Shubina, F. Hampel, K. Meyer, M. Kivala, Angew. Chem. 2016, 128, 13795-13799; T. A. Schaub, E. M. Zolnhofer, D. P. Halter, T. E. Shubina, F. Hampel, K. Meyer, M. Kivala, Angew. Chem. Int. Ed. 2016, 55, 13597-13601.

[9] a) C. S. Oh, J. M. Choi, J. Y. Lee, Adv. Optical Mater. 2016, 4, 1281-1287; b) J. Yoon, S. K. Kim, H. J. Kim, S. Choi, S. W. Jung, H. Lee, J. Y. Kim, D.-W. Yoon, C. W. Han, W.-S. Chae, J. H. Kwon, M. J. Cho, D. H. Choi, Chem. Eur. J. 2020. 26, 1-10; c) J.-Y. Kim, T.-R. Hong, I.-R. Choe, J.-A. Lee, H.-G. Ryu, B.-M. Seo, J.-H. Yang, C.-W. Han, J.-H. Baek, H.-C. Choi, I.-B. Kang, SID 2020 Digest 2020, ISSN 0097-996X/20/4803-0053.

[10] a) J. P. Cheng, Z. Zheng, Tetrahedron letters, 1996, 37, 1457-1460; b) A. Houmam, Chem. Rev. 2008, 108, 2180-2237; c) K. Daasbjerg, J. Chem. Soc. Perkin Trans. 1994, 2, 1275-1277.

[11] a) H. Yang, Q. Liang, C. Han, J. Zhang, H. Xu, Adv. Mater. 2017, 29, 1700553; b) S. Y. Lee, C. Adachi, T. Yasuda, Adv. Mater. 2016, 28, 4626-4631; c) C. Li, C. Duan, C. Han, H. Xu, Adv. Mater. 2018, 30, 1804228; d) S. Gong, Y.-L. Chang, K. Wu, R. White, Z.-H. Lu, D. Song, C. Yang, Chem. Mater. 2014, 26, 1463-1470; e) J. Jia, L. Zhu, Y. Wei, Z. Wu, H. Xu, D. Ding, R. Chen, D. Ma, W. Huang, J. Mater. Chem. C, 2015, 3, 4890-4902.

[12] Z. Liu, F. Cao, T. Tsuboi, Y. Yue, C. Deng, X. Ni, W. Sun, Q. Zhang, J. Mater. Chem. C, 2018, 6, 7728-7733.

[13] S.-G. Ihn, N. Lee, S. O. Jeon, M. Sim, H. Kang, Y. Jung, D. H. Huh, Y. M. Song, S. Y. Lee, M. Numata, H. Miyazaki, R. Gómez-Bombarelli, J. AguileraIparraguirre, T. Hirzel, A. Aspuru-Guzik, S. Kim, S. Lee, Adv. Sci. 2017, 4, 1600502.

[14] D. Zhang, L. Duan, Y. Li, H. Li, Z. Bin, D. Zhang, J. Qiao, G. Dong, L. Wang, Y. Qiu, Adv. Funct. Mater. 2014, 24, 3551-3561.

[15] M. Jung, M. H. Lee, H. Y. Lee, T. Kim, Mater. Horiz., 2020, 7, 559-565.

[16] D. Zhang, P. Wei, D. Zhang, L. Duan, ACS Appl. Mater. Interface 2017, 9, 19040-19047. 